\title{
TRAQUELECTOMÍA RADICAL VAGINAL CON LINFADENECTOMÍA PÉLVICA POR LAPAROSCOPIA PARA CÁNCER DE CÉRVIX TEMPRANO: REPORTE DE DOS CASOS
}

\section{Radical vaginal trachelectomy with laparoscopic pelvic lymphadenectomy for early cervical cancer: a report of two cases}

Jorge Alexánder Rodríguez, M.D. *, José Duván López, M.D.**

Recibido: octubre 4/07 - Aceptado: mayo 14/08.

\section{RESUMEN}

Introducción: el cáncer de cérvix es la neoplasia ginecológica más común en países en desarrollo donde las pacientes con deseo reproductivo no tenían en el pasado posibilidades quirúrgicas de tratar el cáncer y al mismo tiempo conservar la fertilidad. El objetivo de la presentación de estos casos es reportar la factibilidad de realizar el procedimiento en nuestro medio.

Casos clínicos: se presentan dos casos de pacientes con cáncer de cérvix IA2 y IB1, de 29 y 24 años respectivamente, con deseo de conservar la fertilidad, a quienes se les realizó traquelectomía radical vaginal y linfadenectomía pélvica por laparoscopia con buen resultado quirúrgico.

Conclusión: las técnicas de tratamiento mínimamente invasivas en pacientes con cáncer invasor de cérvix surgen como una alternativa para considerar cuando se quiere conservar la fertilidad.

Palabras clave: traquelectomía, laparoscopia, cáncer de cérvix.

* Ginecólogo Oncólogo, Clínica Saludcoop, Medellín, Colombia Correo electrónico: jorgear12@gmail.com

** Fellow de Ginecología endoscópica, Universidad CES, ginecólogo clínica Saludcoop Medellín, Colombia.

\section{SUMMARY}

Introduction: cervical cancer is the most common gynaecological neoplasia in developing countries, where patients wishing to reproduce in the past had no surgical options open to them for treating cancer and preserving their fertility at the same time. This paper was aimed at reporting the feasibility of carrying out the procedure in our setting.

Clinical cases: the cases presented concern two female patients aged 24 and 29 having cervical cancer IA2 and IB1, respectively. They wished to preserve their reproductive functions and underwent radical vaginal trachelectomy and laparoscopic pelvic lymphadenectomy with successful surgical results. Conclusion: minimal invasive treatment techniques in patients suffering from invasive cervical cancer represent an alternative when a patient wants to preserve her fertility.

Key words: trachelectomy, cervical cancer, laparoscopy.

\section{INTRODUCCIÓN}

El cáncer de cérvix es la neoplasia más común en la mujer en edad reproductiva en los países en vía de desarrollo. Aproximadamente el 15\% de las 
pacientes con estadios tempranos tienen deseo de fertilidad. ${ }^{1}$ En el pasado la única alternativa que se ofrecía para los estadios IA2 ó IB1 era el manejo radical sin conservación de la fertilidad, es decir, histerectomía radical más linfadenectomía pélvica o radioterapia. ${ }^{2}$ Sin embargo, desde 1994 se comenzó a realizar en Francia el manejo de estadios IA2 e IB1 con traquelectomía radical vaginal más linfadenectomía pélvica por laparoscopia con resultados oncológicos similares a las técnicas no conservadoras. ${ }^{3}$

El objetivo de la presentación de dos casos de pacientes con cáncer de cérvix temprano, manejados con traquelectomía radical vaginal, linfadenectomía pélvica y biopsia paraaórtica por laparoscopia, es presentar las indicaciones, revisar la técnica quirúrgica y las complicaciones tanto intraoperatorias como posoperatorias.

\section{Técnica quirúrgica}

El procedimiento consiste en realizar la traquelectomía vaginal radical con vaginectomía de por lo menos un centímetro, preservación de las arterias uterinas, resección de parametrios en su tercio medio, lo cual indica la radicalidad y amputación del cuello uterino $5 \mathrm{~mm}$ por debajo del istmo; colocando previo a la cirugía catéteres doble "j" endoureterales y de esta manera evitar la lesión ureteral mediante la palpación de estos durante la disección de los parametrios. Al terminar la traquelectomía se continúa realizando cerclaje con sutura no absorbible, y sutura del itsmo a la vagina para así concluir el tiempo vaginal.

Si no se evidencia compromiso macroscópico de los bordes del cuello, parametrios o vagina se continúa con el tiempo abdominal por laparoscopia, con la realización del puerto supraumbilical de $10 \mathrm{~mm}$, dos laterales derechos de $5 \mathrm{~mm}$ y dos laterales izquierdos de $5 \mathrm{~mm}$, para realizar biopsia paraaórtica, seguido de linfadenectomía pélvica desde la bifurcación de las arterias ilíacas hasta el agujero obturador bilateralmente y de esta manera se concluye el procedimiento. ${ }^{4}$

\section{CASOS CLÍNICOS}

\section{Caso 1}

Paciente de 29 años, soltera, sin hijos, quien se realizó citología de rutina con reporte de lesión intraepitelial de bajo grado. La colposcopia fue satisfactoria con cambios mayores, se toma biopsia que reporta una neoplasia intraepitelial cervical de alto grado (NIC III). Con estos hallazgos se programó una conización cervical de 2,5 cm con reporte de: carcinoma escamocelular microinfiltrante del cérvix que compromete hasta $4 \mathrm{~mm}$ en profundidad y 3 mm en diámetro con bordes libres. En el examen físico se encontró al tacto rectovaginal un cuello de 3 centímetros, los parametrios y vagina libres de tumor; los paraclínicos de extensión pertinentes del caso fueron negativos para metástasis. Con un diagnóstico de cáncer de cérvix estadio IA2, la paciente opta por la traquelectomía radical vaginal y linfadenectomía pélvica por laparoscopia con la intención de conservar su función reproductiva.

Previo a la cirugía se solicitó a urología la colocación de catéter ureteral doble “j”, para palpación de uréteres durante la disección de los parametrios. Se realizó la cirugía en junio del 2007, con un tiempo total de 270 minutos, sin complicaciones intraoperatorias y dada de alta a las 48 horas. La patología reportó: cérvix que mide $3 \mathrm{~cm}$ de diámetro por 2 cm de profundidad sin lesión residual, cervicitis crónica moderada y depósito de tejido de granulación, todos los bordes son negativos. Ganglios linfáticos: 22 ganglios del vaciamiento pélvico sin metástasis incluidos dos paraaórticos.

La paciente presentó linfoquiste en área ilíaca derecha 20 días luego de la cirugía que se resolvió con manejo expectante.

\section{Caso 2}

Paciente de 24 años, casada, un hijo por parto vaginal, se realizó citología de rutina que reportó lesión intraepitelial de bajo grado. Colposcopia satisfactoria con cambios mayores y se tomó biopsia que reportó carcinoma escamocelular con adenocarcinoma infiltrante concomitante. Se le realizó cono cervical 
frío de 2,5 cm de diámetro y $2 \mathrm{~cm}$ de profundidad con reporte de carcinoma escamocelular infiltrante de célula grande, moderadamente diferenciado con compromiso del 20\% del espesor del cérvix, bordes libres de lesión.

En el examen físico se encontró al tacto rectovaginal un cuello de 3 centímetros, los parametrios y vagina libres de tumor y los exámenes de extensión pertinentes para el caso fueron negativos. Con diagnóstico de cáncer de cérvix estadio IB1, la paciente opta por la traquelectomía radical vaginal y linfadenectomía pélvica por laparoscopia, con intención de preservar el útero para futuros embarazos. Se solicitó la colocación de catéter doble “j” ureteral por el servicio de urología. Se realizó el procedimiento en agosto del 2007, con un tiempo total de 300 minutos, sin complicaciones y dada de alta a las 48 horas. La patología reporto: cérvix que mide $2 \times 2 \times 1,5 \mathrm{~cm}$ con un reborde vaginal que sobresale hasta $1 \mathrm{~cm}$ de longitud negativo para carcinoma invasor residual. Focos de displasia escamosa ligera asociada a VPH (virus del papiloma humano) a nivel exocervical; borde de resección cervicovaginal negativo para tumor. 21 ganglios del vaciamiento ganglionar pélvico negativos para metástasis, incluidos tres ganglios paraaórticos. La evolución posoperatoria fue satisfactoria.

\section{DISCUSIÓN}

Desde 1994, el doctor Daniel Dargent de Francia, publicó por primera vez el manejo de pacientes con cáncer de cérvix en estadios iniciales con traquelectomía radical más linfadenectomía pélvica por laparoscopia con excelentes resultados, desde entonces se ha realizado el procedimiento en alrededor de 500 pacientes en el mundo. ${ }^{3}$

Los criterios para que una paciente sea candidata a la cirugía incluyen los estadios IA1 con compromiso linfovascular, IA2 ó IB1 de cáncer de cérvix, deseo de conservar la fertilidad, tumor menor de $2 \mathrm{~cm}$ y ausencia de compromiso linfovascular (en los estadios IA2 e IB1); de igual manera para que la cirugía se considere exitosa debe tener los siguientes criterios: bordes libres, compromiso del estroma cervical menor del 50\% y vaciamiento ganglionar pélvico negativo con más de 12 ganglios, idealmente veinte. $^{4}$

El equipo humano requerido para el procedimiento consta de ginecólogo-oncólogo con experiencia en cirugía endoscópica y ginecólogo endoscopista, ya que se requiere conocimiento tanto en anatomía y cirugía retroperitoneal como en técnicas endoscópicas avanzadas y cirugía vaginal. ${ }^{4}$ Sin embargo, en otros países el procedimiento es realizado en su totalidad por ginecólogos oncólogos, todo depende de la formación de los diferentes centros oncológicos. ${ }^{5}$ En cuanto a los requerimientos tecnológicos y de instrumental, se necesita torre completa de laparoscopia, energía mono y bipolar, instrumental completo de cirugía vaginal y el recurso de interconsulta por urología para la inserción de los catéteres doble “j”, endoureterales. De ser posible es ideal contar con bisturí armónico endoscópico, que permite mayor seguridad en la disección de tejidos.

El tiempo promedio de la cirugía fue de 285 minutos, el cual está acorde con el reporte de Hertman y colaboradores en su trabajo prospectivo de 100 pacientes llevadas a traquelectomía radical, en el cual se presentó un promedio de 253 (115-402) minutos. ${ }^{6}$ El sangrado durante el procedimiento se ha reportado entre 50 y $600 \mathrm{ml}$ con un promedio de $203 \mathrm{ml}^{5}$

Las complicaciones intraoperatorias reportadas incluyen sangrado mayor con necesidad de transfusión (6\%), lesión de vejiga (2\%) y linfoquistes (1\%), así como posoperatorias: linfedema en un caso, disfunción vesical que se recupera en todos los casos entre 10 y 50 días y estenosis cervical. ${ }^{4-6}$

En los diferentes reportes el pronóstico oncológico ha sido similar a la cirugía radical no conservadora con sobrevida mayor al $90 \%$ a cinco años y recaídas entre el 2 y 4\%, lo cual ha permitido el desarrollo de la técnica a nivel mundial con buena aceptación. ${ }^{7,8}$ En cuanto a los resultados reproductivos, como es de esperar con esta técnica en donde 
la zona residual de cuello es menor de $0,5 \mathrm{~cm}$, la incompetencia cervical es causante de una tasa del $16 \%$ de pérdidas en el primer trimestre, y de $8 \%$ en el segundo trimestre, con un 20\% de partos prematuros. ${ }^{9}$ Sin embargo, el éxito reproductivo entendido como bebé sano en casa es del 70\% dentro del grupo de mujeres que deciden embarazarse, lo cual es bastante alentador si se parte de la base de que en el pasado estas pacientes no tenían ninguna posibilidad de tener hijos luego del tratamiento convencional, de tal manera que a la fecha se han reportado alrededor de 100 cesáreas con feto vivo. ${ }^{10,11}$

\section{CONCLUSIÓN}

La técnica mínimamente invasiva en pacientes con cáncer invasor, estadios IA2 e IB1 de cérvix, surgen como una alternativa para considerar, cuando se quiere conservar la fertilidad.

\section{REFERENCIAS}

1. Van der Vange N, Weverling G, Ketting B, Ankum WM, Samlal R, Lammus FB. The prognosis of cervical cancer associated with pregnancy: a matched cohort study. Obstet Gynecol 1995;85:1022-6.

2. DiSaia PJ, Creasman WT. Invasive Cancer Cervical. En: DiSaia PJ, Creasman WT, editors. Clinical gynecologic oncology. 5th ed. St. Louis: Mosby Year Book; 1997. p. 51-100.

3. Dargent D, Brun JL, Roy M, Remi I. Pregnancies following radical trachelectomy for invasive cervical cancer. Gynecol Oncol 1994;52:105.

4. Dargent D, Martin X, Sacchettoni A, Mathevet P.
Laparoscopic vaginal radical trachelectomy: a treatment to preserve the fertility of cervical carcinoma patients. Cancer 2000;88:1877-82.

5. Schlaerth JB, Spirtos NM, Schlaerth AC. Radical trachelectomy and pelvic lymphadenectomy with uterine preservation in the treatment of cervical cancer. Am J Obstet Gynecol 2003;188:29-34.

6. Hertel H, Köhler C, Grund D, Hillemanns P, Possover M, Michels W, Schneider A. Radical vaginal trachelectomy (RVT) combined with laparoscopic pelvic lymphadenectomy: prospective multicenter study of 100 patients with early cervical cancer. Gynecol Oncol 2006;103:506-11.

7. Morice P, Dargent D, Haie-Meder C, Duvillard P, Castaigne D. First case of a centropelvic recurrence after radical trachelectomy: literature review and implications for the preoperative selection of patients. Gynecol Oncol 2004;92:1002-5.

8. Covens A, Shaw P, Murphy J, DePetrillo D, Lickrish $\mathrm{G}$, Laframboise $\mathrm{S}$, et al. Is radical trachelectomy a safe alternative to radical hysterectomy for patients with stage IA-B carcinoma of the cervix? Cancer 1999;86:2273-9.

9. Plante M, Renaud MC, Francois H, Roy M. Vaginal radical trachelectomy: an oncologically safe fertilitypreserving surgery. An updated series of 72 cases and review of the literature. Gynecol Oncol 2004; 94:61423.

10. Boss EA, van Golde RJ, Beerendonck CC, Massuger LF, Pregnancy after radical trachelectomy: a real option? Gynecol Oncol 2005;99:S152-6.

11. Plante M, Renaud MC, Hoskins IA, Roy M. Vaginal radical trachelectomy: a valuable fertility-preserving option in the management of early-stage cervical cancer. A series of 50 pregnancies and review of the literature. Gynecol Oncol 2005;98:3-10. 